\title{
Synthesis and Antimicrobial Activity of Some Novel Pyrazolones
}

\author{
S. D. MHASKE', S. J. TAKATE', R. N. DHAWALE², \\ H. N. AKOLKAR ${ }^{1}$, P. V. RANDHAVANE ${ }^{1}$ and B. K. KARALE ${ }^{1 *}$ \\ ${ }^{1}$ Department of Chemistry, Radhabai Kale Mahila Mahavidyalaya, Ahmednagar, 414001, India. \\ 2Department of Chemistry, New Arts, Commerce \& Science College, Ahmednagar, 414001, India. \\ ${ }^{*}$ Corresponding author E-mail: bkkarale @ yahoo.com \\ http://dx.doi.org/10.13005/ojc/330330
}

(Received: February 28, 2017; Accepted: April 16, 2017)

\begin{abstract}
Knoevenagel condensation was carried out using heterocyclic aldehydes and pyrazolone derivatives. The structure elucidation of condensation products 1,2, 3 and 4 was done using spectral methods like IR, ${ }^{1} \mathrm{H}$ NMR and mass spectrometry. These novel compounds were screened for antimicrobial activity.
\end{abstract}

Keywords: Pyrazolone, Knoevenagel condensation, Antimicrobial activity.

\section{INTRODUCTION}

Multi drug resistant micro-organisms and increased systemic as well as infectious diseases are the two major challenges for scientific world. Development of newer synthetic entities can offer a major solution for these problems.

Pyrazolone containing compounds are associated with antimicrobial ${ }^{1}$, antiviral ${ }^{2}$, antifungal ${ }^{3}$, antioxidant ${ }^{4}$, cytotoxic ${ }^{5}$, analgesic ${ }^{6}$, anti-inflammatory ${ }^{7}$ activities. Thiophene derivatives have found very important place in the field of drug discovery because of their potential biological activities $^{8}$.
Thiazoles have found applications in drug development for treatment of HIV infections ${ }^{9}$, hypertension ${ }^{10}$ and as inhibitors of bacterial gyrase $\mathrm{B}^{11}$. Moreover pyrazole containing compounds are reported to have good biological activities like antimicrobial ${ }^{12}$, antifungal ${ }^{13}$, antiviral ${ }^{14}$, analgessic ${ }^{15}$,anti parastic ${ }^{16}$ and antineoplastic ${ }^{17}$.

Pyridine nucleus is important nitrogen heterocycle which exhibits antimicrobial ${ }^{18}$, anticancer ${ }^{19}$ and antioxidant ${ }^{20}$ activities. Imidazole derivatives have been shown to exhibit antibacterial ${ }^{21}$, antifungal and antioxidant ${ }^{22}$ activity. 
It has been shown that substitution pattern of bromine can alter the activity of a compound ${ }^{23}$. Fluorine containing compounds are associated with bioactivities such as antibacterial ${ }^{24}$, anticancer ${ }^{25}$ and analgesic ${ }^{26}$.

Knoevenagel condensation ${ }^{27}$ reaction shave occupied an important place in synthetic organic chemistry due to its efficiency in carboncarbon bond formation. Many researchers have studied Knoevenagel condensation reactions of various aldehydes with pyrazolones ${ }^{28-29}$.

Activities associated with pyrazolones, thiophenes, pyrazoles, imidazoles and halogens prompted us to synthesize compound containing these moieties.

\section{EXPERIMENTAL}

Physical constants were determined in open capillary. IR of the compounds were recorded using IR Affinity-1 Fourier transform infrared spectrophotometer (Shimadzu). PMR spectra were taken using Bruker Avance II $400 \mathrm{MHz}$ NMR spectrometer. TMS was used as an internal standard and DMSO- $d_{6}$ as a solvent. The mass spectra were recorded on HP 1100 LC/MS.

(4E)-4-((3-(5-Bromothiophen-2-yl)-1-(2,3dimethylphenyl)-1 $H$ - pyrazol-4-yl) methylene)-1(2,3-dimethylphenyl)-3-propyl-1 H-pyrazol-5(4H)one, 1.

The mixture of 3-(5-bromothiophen-2-yl)-1(2,3-dimethylphenyl)-1 $\mathrm{H}$ - pyrazole-4-carbaldehyde A (0.01 mol) and 2-(2,3-dimethylphenyl)-5-propyl2,4-dihydro-3H-pyrazol-3-one $B(0.01 \mathrm{~mol})$ in $5 \mathrm{~mL}$ of glacial acetic acid was refluxed in round bottom flask for $30 \mathrm{~min}$. Reaction progress was studied and confirmed using TLC at the end of reaction, reaction mixture was poured over ice. Product obtained 1 was separated using simple filtration then purified by re crystallization from ethanol. Compounds 2,3 and 4 were synthesized using same procedure. Physical data of all the synthesized compounds is given in Table 1 and spectral data as below.<smiles>[R]C1=NN([Al])C(=O)C1=Cc1cn(-c2cccc(C)c2C)nc1-c1ccc(Br)s1</smiles><smiles>[R]C1=NN([Al])C(=O)C1=Cc1c(Cl)nc(CCCC)n1Cc1nccc(OCC(F)(F)F)c1C</smiles>

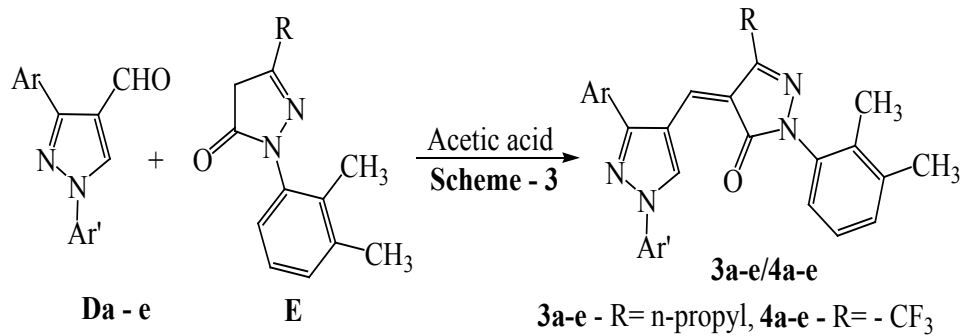


MHASKE et al., Orient. J. Chem., Vol. 33(3), 1304-1310 (2017)

1306

Table 1: Physical data of synthesized compounds

\begin{tabular}{llrlrrr}
\hline Comp & Structure & M.P. & $\%$ & Compd & Structure & M. P. \\
$\left({ }^{\circ} \mathrm{C}\right)$ & Yield & & $\begin{array}{r}\% \\
\left({ }^{\circ} \mathrm{C}\right)\end{array}$ & Yield \\
\hline
\end{tabular}

$1 \mathrm{a}$

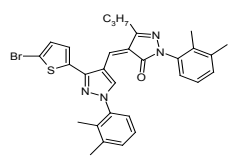

$1 b$

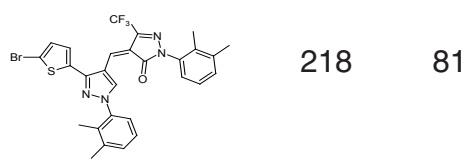

$1 c$

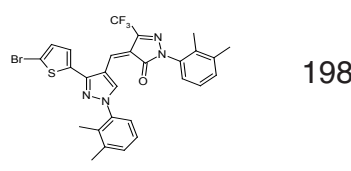

$1 d$

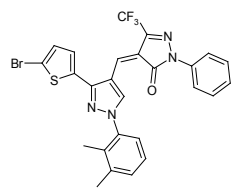

$1 e$

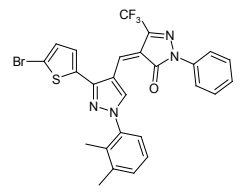

$1 f$

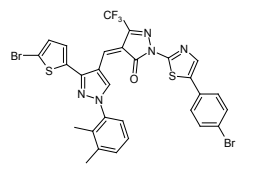

$1 \mathrm{~g}$

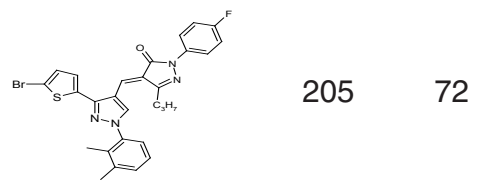

$1 \mathrm{~h}$

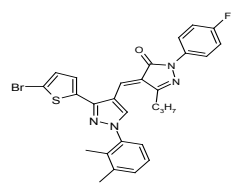

$2 a$
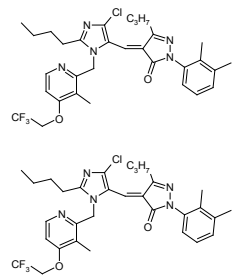

235

77

74

223

83

258

75

$227 \quad 82$

224

84

135

60

140

66
$2 f$

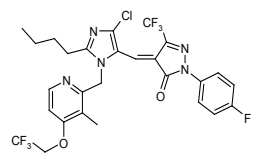

$2 g$

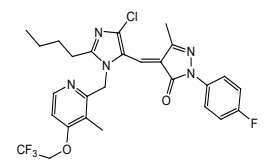

$3 b$

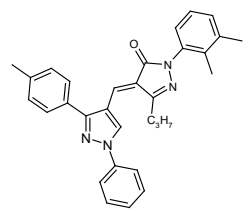

$3 c$

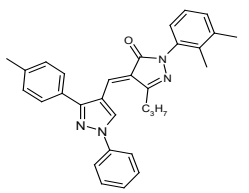

$4 b$

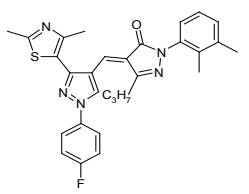

$3 d$

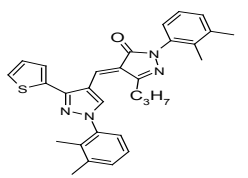

$3 e$

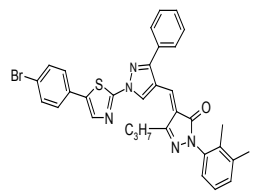

$4 a$
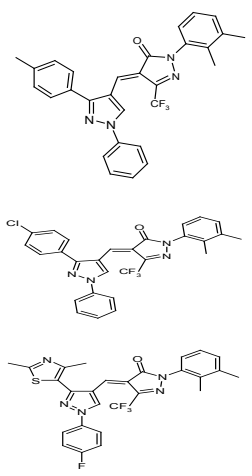

$4 c$
166

64

180

62

188

60

178

63

205

61

210

65

245

70

204

72

228

71 


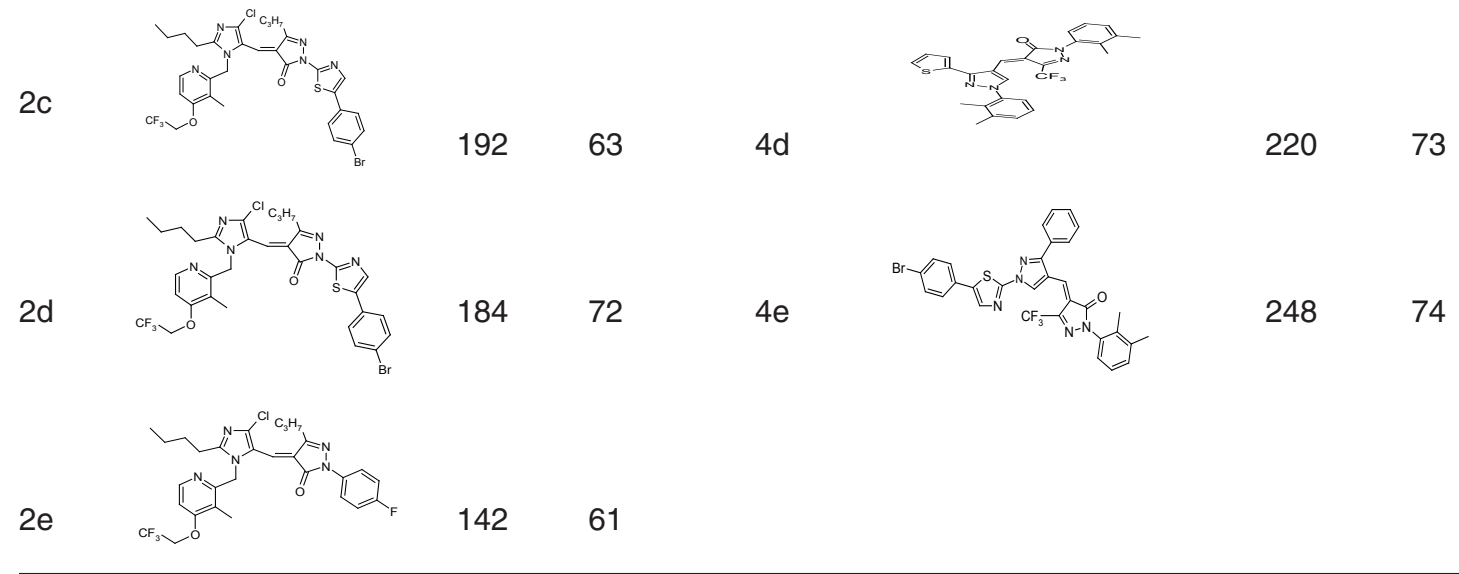

1a, MS: $m / z 572\left(\mathrm{M}^{+}\right)$; PMR: $\delta 9.74(\mathrm{~s}$, $1 \mathrm{H}), 7.70(\mathrm{~s}, 1 \mathrm{H}), 7.37(\mathrm{~d}, 1 \mathrm{H}, J=3.8 \mathrm{~Hz}), 7.32(\mathrm{~d}, 2 \mathrm{H}, J$ $=3.8 \mathrm{~Hz}), 7.25-7.26(\mathrm{~m}, 2 \mathrm{H}), 7.11-7.17(\mathrm{~m}, 3 \mathrm{H}), 2.68$ $(\mathrm{t}, 2 \mathrm{H}), 2.36(\mathrm{~s}, 3 \mathrm{H}), 2.31(\mathrm{~s}, 3 \mathrm{H}), 2.12(\mathrm{~s}, 3 \mathrm{H}), 2.08$ (s, 3H), 1.77 (sextet, 2H), 1.04 (t, 3H); IR: 3132, 2958, 2918, 1676, 1554, 790, 754, 717, $663 \mathrm{~cm}^{-1}$.

1b, MS: m/z $598\left(\mathrm{M}^{+}\right)$; PMR: $\delta 9.76(\mathrm{~s}, 1 \mathrm{H})$, $7.81(\mathrm{~s}, 1 \mathrm{H}), 7.42(\mathrm{~d}, 1 \mathrm{H}, J=4 \mathrm{~Hz}), 7.34(\mathrm{~d}, 1 \mathrm{H}, J=$ $4.8 \mathrm{~Hz}), 7.27-7.31(\mathrm{~m}, 4 \mathrm{H}), 7.18(\mathrm{t}, 2 \mathrm{H}, J=7 \mathrm{~Hz}), 2.35$ (s, 3H),2.32 (s, 3H), $2.11(\mathrm{~s}, 3 \mathrm{H}), 2.06$ (s, 3H); IR: 3122, 2920, 1691, 1593, 1112, 794, 754, 713, 619 $\mathrm{cm}^{-1}$.

1c, MS: $m / z 544\left(\mathrm{M}^{+}\right)$; PMR: $\delta 9.71(\mathrm{~s}$, $1 \mathrm{H}), 7.84-7.93(\mathrm{~m}, 5 \mathrm{H}), 7.66(\mathrm{~s}, 1 \mathrm{H}), 7.56-7.58(\mathrm{~m}, 3 \mathrm{H})$, $7.42(\mathrm{~d}, 1 \mathrm{H}, J=3.9 \mathrm{~Hz}), 7.33(\mathrm{~d}, 1 \mathrm{H}, J=3.9 \mathrm{~Hz}), 2.80$ (t, $2 \mathrm{H}), 2.33$ (s, 3H), 2.15 (s, 3H), 1.81 (sextet, $2 \mathrm{H}$ ), 1.08 (t, 3H); IR: 3128, 2952, 2911, 1669, 1544, 792, $758,719 \mathrm{~cm}^{-1}$.

1d, MS: m/z $570\left(\mathrm{M}^{+}\right)$; PMR: $\delta 9.82(\mathrm{~s}$, $1 \mathrm{H}), 8.69(\mathrm{~s}, 1 \mathrm{H}), 7.87-8.09(\mathrm{~m}, 5 \mathrm{H}), 7.51-7.54(\mathrm{~m}$, $3 \mathrm{H}), 7.42(\mathrm{~d}, 1 \mathrm{H}, J=3.8 \mathrm{~Hz}), 7.33(\mathrm{~d}, 1 \mathrm{H}, J=3.8$ $\mathrm{Hz}), 2.38$ (s, 3H), 2.12 (s, 3H); IR: 3122, 2925, 1693, $1597,1120,790,717,688 \mathrm{~cm}^{-1}$.

1e, MS: $m / z 707\left(\mathrm{M}^{+}\right)$; PMR: $\delta 9.76(\mathrm{~s}, 1 \mathrm{H})$, $7.93(\mathrm{~d}, 2 \mathrm{H}, \mathrm{J}=7.8 \mathrm{~Hz}), 7.70(\mathrm{~s}, 1 \mathrm{H}), 7.36-7.41(\mathrm{~m}$, 4H), 7.31-7.33 (m, 3H),7.16 (t, 1H), $2.71(\mathrm{t}, 2 \mathrm{H}, J$ $=7.4 \mathrm{~Hz}$ ), $2.39(\mathrm{~s}, 3 \mathrm{H}), 2.14(\mathrm{~s}, 3 \mathrm{H}), 1.81$ (sextet, $2 \mathrm{H}$, $J=7.4 \mathrm{~Hz}$ ), 1.07 (t, 3H, J=7.4 Hz); IR: 3128, 2926, $1693,1597,1120,790,756,715,629 \mathrm{~cm}^{-1}$.

1f, MS: $m / z 731\left(\mathrm{M}^{+}\right)$; PMR: $\delta 9.79(\mathrm{~s}, 1 \mathrm{H})$, 7.79 (s, 1H), 7.85 (d, $2 \mathrm{H}, J=7.7 \mathrm{~Hz}), 7.49$ (d, 2H,
$J=7.7 \mathrm{~Hz}), 7.42(\mathrm{~d}, 1 \mathrm{H}, J=3.88 \mathrm{~Hz}), 7.30-7.35(\mathrm{~m}$, $5 \mathrm{H}), 2.38$ (s, 3H),2.14 (s, 3H); IR : 3127, 2924, 1697, $1596,1117,793,757,718,639 \mathrm{~cm}^{-1}$.

1g, MS: m/z $562\left(\mathrm{M}^{+}\right)$; PMR: $\delta 9.73(\mathrm{~s}, 1 \mathrm{H})$, $7.93(\mathrm{dd}, 2 \mathrm{H}, J=9.2 \& 5 \mathrm{~Hz}), 7.73(\mathrm{~s}, 1 \mathrm{H}), 7.44(\mathrm{~d}, 1 \mathrm{H}$, $J=3.8 \mathrm{~Hz}), 7.37-7.39(\mathrm{~m}, 2 \mathrm{H}), 7.30-7.33(\mathrm{~m}, 2 \mathrm{H}), 7.22$ $(\mathrm{t}, 2 \mathrm{H}, J=8.8 \mathrm{~Hz}), 2.72(\mathrm{t}, 2 \mathrm{H}), 2.38(\mathrm{~s}, 3 \mathrm{H}), 2.12$ (s, 3H),1.78 (sextet, 2H, J=7.3 Hz), 1.04 (t, 3H); IR : 3135, 2968, 2922, 1671, 1559, 788, 757, 722, $687 \mathrm{~cm}^{-1}$.

1h, MS: m/z $588\left(\mathrm{M}^{+}\right)$; PMR: $\delta 9.82(\mathrm{~s}, 1 \mathrm{H})$, 7.88 (dd, $2 \mathrm{H}, J=9.12 \& 4.92 \mathrm{~Hz}), 7.75(\mathrm{~s}, 1 \mathrm{H}), 7.45$ $(\mathrm{d}, 1 \mathrm{H}, J=3.88 \mathrm{~Hz}), 7.36-7.39(\mathrm{~m}, 2 \mathrm{H}), 7.32-7.35$ $(\mathrm{m}, 2 \mathrm{H}), 7.22(\mathrm{t}, 2 \mathrm{H}, J=8.8 \mathrm{~Hz}), 2.38(\mathrm{~s}, 3 \mathrm{H}), 2.12$ (s, 3H); IR: 3133, 2929, 1690, 1593,1123, 798, 755, $722,689 \mathrm{~cm}^{-1}$.

2a, MS: $m / z 601\left(\mathrm{M}^{+}\right)$; PMR: $\delta 8.18(\mathrm{~d}, 1 \mathrm{H}$, $J=5.7 \mathrm{~Hz}), 7.48(\mathrm{~s}, 1 \mathrm{H}), 7.45(\mathrm{t}, 1 \mathrm{H}, J=8 \mathrm{~Hz}), 7.09$ $7.18(\mathrm{~m}, 2 \mathrm{H}), 7.02(\mathrm{~d}, 1 \mathrm{H}, J=5.7 \mathrm{~Hz}), 5.46(\mathrm{~s}, 2 \mathrm{H}), 4.81$ $(\mathrm{q}, 2 \mathrm{H}), 2.67(\mathrm{t}, 2 \mathrm{H}), 2.61(\mathrm{t}, 2 \mathrm{H}), 2.33(\mathrm{~s}, 3 \mathrm{H}), 2.22$ $(\mathrm{t}, 3 \mathrm{H}), 2.12$ (t, 3H),1.78 (sextet, 2H),1.59(quintet, $2 \mathrm{H}), 1.32$ (sextet, 2H), $1.05(\mathrm{t}, 3 \mathrm{H}), 0.84(\mathrm{t}, 3 \mathrm{H}$, $J=7.32 \mathrm{~Hz}$ ); IR:2928, 1656, 1623, 1558, 1234, 1126 , $967,769,754,711 \mathrm{~cm}^{-1}$.

2b, MS: $m / z 627\left(\mathrm{M}^{+}\right)$; PMR: $\delta 8.16(\mathrm{~d}, 1 \mathrm{H}$, $J=5.7 \mathrm{~Hz}), 7.50(\mathrm{~s}, 1 \mathrm{H}), 7.43(\mathrm{t}, 1 \mathrm{H}, J=8 \mathrm{~Hz}), 7.09$ $7.20(\mathrm{~m}, 2 \mathrm{H}), 7.04(\mathrm{~d}, 1 \mathrm{H}, J=5.7 \mathrm{~Hz}), 5.45(\mathrm{~s}, 2 \mathrm{H}), 4.80$ $(\mathrm{q}, 2 \mathrm{H}), 2.62(\mathrm{t}, 2 \mathrm{H}), 2.32(\mathrm{t}, 3 \mathrm{H}), 2.21(\mathrm{t}, 3 \mathrm{H}), 2.14$ $(\mathrm{t}, 3 \mathrm{H}), 1.57$ (quintet, $2 \mathrm{H}$ ), 1.33 (sextet, $2 \mathrm{H}$ ), 0.85 (t, 3H, J=7.4 Hz); IR: 2958, 2920, 1655, 1620, 1524, $1231,1120,967,788 \mathrm{~cm}^{-1}$ 
2c, MS: $m / z 734\left(\mathrm{M}^{+}\right)$; PMR: $\delta 8.20(\mathrm{~d}, 1 \mathrm{H}, \mathrm{J}=$ $5.7 \mathrm{~Hz}), 8.07(\mathrm{~s}, 1 \mathrm{H}), 7.93(\mathrm{~d}, 2 \mathrm{H}, J=7.7 \mathrm{~Hz}), 7.50$ (d, $2 \mathrm{H}, J=5.8 \mathrm{~Hz}), 7.47(\mathrm{~s}, 1 \mathrm{H}), 7.05(\mathrm{~d}, 1 \mathrm{H}, J=5.7 \mathrm{~Hz}), 5.47$ $(\mathrm{s}, 2 \mathrm{H}), 4.80(\mathrm{q}, 2 \mathrm{H}), 2.68(\mathrm{t}, 2 \mathrm{H}), 2.62(\mathrm{t}, 2 \mathrm{H}), 2.24$ (s, 3H), 1.77 (sextet, 2H), 1.60 (quintet, 2H), 1.34 (sextet, 2H), 1.07 (t, 3H), $0.87(\mathrm{t}, 3 \mathrm{H}, J=7.4 \mathrm{~Hz}$ ); IR: 2927, 2918, 1676, 1622, 1525, 1231, 1123, $966 \mathrm{~cm}^{-1}$.

2d, MS: $m / z 760\left(\mathrm{M}^{+}\right)$; PMR: $\delta 8.16(\mathrm{~d}, 1 \mathrm{H}$, $J=5.7 \mathrm{~Hz}), 8.06(\mathrm{~s}, 1 \mathrm{H}), 7.91(\mathrm{~d}, 2 \mathrm{H}, J=7.8 \mathrm{~Hz}), 7.49$ (d, 2H, J=7.8 Hz),7.48 (s, 1H),7.05 (d, 1H, J=5.7 $\mathrm{Hz}$ ),5.48 (s, 2H),4.81 (q, 2H),2.63 (t, 2H),2.22 $(\mathrm{t}, 3 \mathrm{H}), 1.62$ (quintet, $2 \mathrm{H}$ ), 1.32 (sextet, $2 \mathrm{H}$ ), 0.88 (t, 3H, J=7.3 Hz); IR: 2925, 2916, 1652, 1626, 1554, $790,1233,1126,963,755 \mathrm{~cm}^{-1}$.

2e, MS: $m / z 591\left(\mathrm{M}^{+}\right)$; PMR: $\delta 8.20(\mathrm{~d}, 1 \mathrm{H}$, $J=5.5 \mathrm{~Hz}), 7.85-7.91(\mathrm{~m}, 2 \mathrm{H}), 7.48(\mathrm{~s}, 1 \mathrm{H}), 7.20$ $(\mathrm{t}, 2 \mathrm{H}, J=8.8 \mathrm{~Hz}), 7.03(\mathrm{~d}, 1 \mathrm{H}, J=5.5 \mathrm{~Hz}), 5.47$ $(\mathrm{s}, 2 \mathrm{H}), 4.78(\mathrm{q}, 2 \mathrm{H}), 2.66(\mathrm{t}, 2 \mathrm{H}), 2.62(\mathrm{t}, 2 \mathrm{H}), 2.21$ $(\mathrm{t}, 3 \mathrm{H}), 1.76$ (sextet, 2H),1.57 (quintet, 2H),1.33 (sextet, 2H), 1.05 (t, 3H), 0.84 (t, 3H, J=7.4 Hz); IR: 2928, 2915, 1656, 1622, 1524, 1230, 1123, 966, $786 \mathrm{~cm}^{-1}$.

2f, MS: m/z $617\left(\mathrm{M}^{+}\right)$; PMR: $\delta 8.19(\mathrm{~d}, 1 \mathrm{H}$, $J=5.5 \mathrm{~Hz}), 7.84-7.90(\mathrm{~m}, 2 \mathrm{H}), 7.47(\mathrm{~s}, 1 \mathrm{H}), 7.19(\mathrm{t}, 2 \mathrm{H}$, $J=8.76 \mathrm{~Hz}), 7.01(\mathrm{~d}, 1 \mathrm{H}, J=5.6 \mathrm{~Hz}), 5.47(\mathrm{~s}, 2 \mathrm{H}), 4.83$ $(\mathrm{q}, 2 \mathrm{H}), 2.63(\mathrm{t}, 2 \mathrm{H}), 2.21(\mathrm{~s}, 3 \mathrm{H}), 1.60$ (quintet, 2H), 1.32 (sextet, $2 \mathrm{H}$ ), 0.85 (t, 3H, J= $7.34 \mathrm{~Hz}$ ); IR: 2938, 2913, 1666, 1622, 1521, 1233, 1120, 966, $789 \mathrm{~cm}^{-1}$.

2g, MS: $m / 2563\left(\mathrm{M}^{+}\right)$; PMR: $\delta 8.18(\mathrm{~d}, 1 \mathrm{H}$, $J=5.6 \mathrm{~Hz}), 7.85-7.89(\mathrm{~m}, 2 \mathrm{H}), 7.45(\mathrm{~s}, 1 \mathrm{H}), 7.19$ (t, $2 \mathrm{H}, J=8.76 \mathrm{~Hz}), 7.03(\mathrm{~d}, 1 \mathrm{H}, J=5.7 \mathrm{~Hz}), 5.49$ $(\mathrm{s}, 2 \mathrm{H}), 4.81(\mathrm{q}, 2 \mathrm{H}, J=8.52 \mathrm{~Hz}), 2.61(\mathrm{t}, 2 \mathrm{H}), 2.21$ (s, 6H), 1.58 (quintet, 2H), 1.30 (sextet, 2H), 0.84 (t, 3H, $J=7.32 \mathrm{~Hz}$ ); IR:2922, 2918, 1651, 1622, 1523, 1230, 1122, $964,781 \mathrm{~cm}^{-1}$.

3a, MS: m/z $474\left(\mathrm{M}^{+}\right)$; PMR: $\delta 10.21(\mathrm{~s}, 1 \mathrm{H})$, 7.89-7.92 (m, 2H), $7.83(\mathrm{~m}, 1 \mathrm{H}), 7.57-7.61(\mathrm{~m}, 4 \mathrm{H})$, 7.42-7.47 (m, 3H), $7.32(\mathrm{~d}, 1 \mathrm{H}, J=7 \mathrm{~Hz}), 7.21-7.24$ $(\mathrm{m}, 2 \mathrm{H}), 2.68(\mathrm{t}, 2 \mathrm{H}), 2.45(\mathrm{~s}, 3 \mathrm{H}), 2.34(\mathrm{~s}, 3 \mathrm{H}), 2.08$ (s, 3H), 1.77 (q, 2H), 1.04 (t, 3H); IR: 3136, 2972, 2929, 2872, 1678, 1598, 1508, 1500, $763 \mathrm{~cm}^{-1}$. 3b, MS: m/z $494\left(\mathrm{M}^{+}\right)$; PMR: $\delta 9.78(\mathrm{~s}, 1 \mathrm{H})$, $7.91(\mathrm{~d}, 2 \mathrm{H}, \mathrm{J}=7.5 \mathrm{~Hz}), 7.70-7.75(\mathrm{~m}, 2 \mathrm{H}), 7.60-7.63$ $(\mathrm{m}, 2 \mathrm{H}), 7.58(\mathrm{t}, 2 \mathrm{H}, J=8 \mathrm{~Hz}), 7.56(\mathrm{~s}, 1 \mathrm{H}), 7.46$ (t, $1 \mathrm{H}, J=7.5 \mathrm{~Hz}), 7.30$ (d, 1H, J = $7 \mathrm{~Hz}), 7.18-7.24$ $(\mathrm{m}, 2 \mathrm{H}), 2.72(\mathrm{t}, 2 \mathrm{H}), 2.35(\mathrm{~s}, 3 \mathrm{H}), 2.09(\mathrm{~s}, 3 \mathrm{H}), 1.72$ (sextet, 2H), 1.09 (t, 3H); IR: 3125, 2926, 1697, 1588, $1508,1503,1165,835,748 \mathrm{~cm}^{-1}$.

3c, MS: m/z $515\left(\mathrm{M}^{+}\right)$; PMR: $\delta 9.43(\mathrm{~s}, 1 \mathrm{H})$, 7.89-7.95 (m, 2H), $7.50(\mathrm{~s}, 1 \mathrm{H}), 7.27-7.43(\mathrm{~m}, 5 \mathrm{H})$, $2.72(\mathrm{~s}, 3 \mathrm{H}), 2.70(\mathrm{t}, 2 \mathrm{H}), 2.43(\mathrm{~s}, 3 \mathrm{H}), 2.35(\mathrm{~s}, 3 \mathrm{H})$, 2.06 (s, 3H), 1.76 (sextet, 2H), 1.02 (t, 3H); IR: 3120, 2922, 1695, 1598, 1508, 1500, 1155,833, 738 $\mathrm{cm}^{-1}$.

3d, MS:m/z $494\left(\mathrm{M}^{+}\right)$; PMR: $\delta 9.74(\mathrm{~s}, 1 \mathrm{H})$, $7.78(\mathrm{~s}, 1 \mathrm{H}), 7.72(\mathrm{dd}, 1 \mathrm{H}, J=1.0 \& 5.1 \mathrm{~Hz}), 7.57(\mathrm{dd}, 1 \mathrm{H}$, $J=1.0 \& 3.6 \mathrm{~Hz}), 7.34(\mathrm{dd}, 1 \mathrm{H}, J=1.4 \& 7 \mathrm{~Hz}), 7.23-$ $7.30(\mathrm{~m}, 3 \mathrm{H}), 7.08-7.19(\mathrm{~m}, 3 \mathrm{H}), 2.66(\mathrm{t}, 2 \mathrm{H}, \mathrm{J}=7.4$ $\mathrm{Hz}), 2.34(\mathrm{~s}, 3 \mathrm{H}), 2.29(\mathrm{~s}, 3 \mathrm{H}), 2.11(\mathrm{~s}, 3 \mathrm{H}), 2.06$ (s, 3H), 1.75 (sextet, 2H, J = 7.4 Hz),1.01 (t, 3H, $J=7.4$ Hz); IR: 3128, 2958, 1681, 1610, 1502, 786, $717 \mathrm{~cm}^{-1}$.

3e, MS: $m / z 621\left(\mathrm{M}^{+}\right)$; PMR: $\delta 10.41$ (s, $1 \mathrm{H}), 8.14(\mathrm{~s}, 1 \mathrm{H}), 7.94(\mathrm{~d}, 2 \mathrm{H}, J=8.5 \mathrm{~Hz}), 7.78$ $(\mathrm{s}, 1 \mathrm{H}), 7.68-7.70(\mathrm{~m}, 2 \mathrm{H}), 7.63-7.66(\mathrm{~m}, 5 \mathrm{H}), 7.36$ $(\mathrm{d}, 1 \mathrm{H}, J=7 \mathrm{~Hz}), 7.21-7.27(\mathrm{~m}, 2 \mathrm{H}), 2.66(\mathrm{t}, 2 \mathrm{H}$, $J=7.4 \mathrm{~Hz}$ ), $2.38(\mathrm{~s}, 3 \mathrm{H}), 2.11(\mathrm{~s}, 3 \mathrm{H}), 1.75$ (sextet, $2 \mathrm{H}$, $J=7.4 \mathrm{~Hz}$ ), 1.01 (t, 3H, $J=7.4 \mathrm{~Hz}$ ); IR: 3132, 2925, 1678, 1600, 1596, 1130, 814, $758 \mathrm{~cm}^{-1}$.

4a, MS: $m / z 500\left(\mathrm{M}^{+}\right)$; PMR: $\delta 10.23(\mathrm{~s}$, $1 \mathrm{H}), 7.90-7.92(\mathrm{~m}, 2 \mathrm{H}), 7.82(\mathrm{~s}, 1 \mathrm{H}), 7.56-7.60$ $(\mathrm{m}, 4 \mathrm{H}), 7.41-7.47(\mathrm{~m}, 3 \mathrm{H}), 7.30(\mathrm{~d}, 1 \mathrm{H}, J=7$ $\mathrm{Hz}), 7.19-7.26(\mathrm{~m}, 2 \mathrm{H}), 2.45(\mathrm{~s}, 3 \mathrm{H}), 2.35(\mathrm{~s}, 3 \mathrm{H}), 2.09$ (s, 3H); IR : 3134, 2920, 1687, 1598, 1498, 1122 , $839 \mathrm{~cm}^{-1}$.

4b, MS: $m / z 520\left(\mathrm{M}^{+}\right)$; PMR: $\delta 10.22$ (s, $1 \mathrm{H}), 7.91(\mathrm{~d}, 2 \mathrm{H}, J=7.5 \mathrm{~Hz}), 7.75(\mathrm{~s}, 1 \mathrm{H}), 7.71-7.74$ $(\mathrm{m}, 2 \mathrm{H}), 7.65-7.67(\mathrm{~m}, 3 \mathrm{H}), 7.58(\mathrm{t}, 2 \mathrm{H}, J=8 \mathrm{~Hz}), 7.45$ $(\mathrm{t}, 1 \mathrm{H}, J=7.5 \mathrm{~Hz}), 7.18-7.25(\mathrm{~m}, 2 \mathrm{H}), 2.34(\mathrm{~s}, 3 \mathrm{H}), 2.08$ (s, 3H); IR: 3120, 2922, 1687, 1602, 1496, 1124, $840,756 \mathrm{~cm}^{-1}$.

4c, MS: $m / z 541\left(\mathrm{M}^{+}\right)$; PMR: $\delta 9.46(\mathrm{~s}$, $1 \mathrm{H}), 7.89-7.95(\mathrm{~m}, 2 \mathrm{H}), 7.50(\mathrm{~s}, 1 \mathrm{H}), 7.26-7.45(\mathrm{~m}, 5 \mathrm{H})$, $2.71(\mathrm{~s}, 3 \mathrm{H}), 2.43$ (s, 3H), 2.36 (s, 3H), 2.08 (s, 3H); IR: 3120, 2916, 2848, 1693, 1600, 1155, 1124, 833, $738 \mathrm{~cm}^{-1}$. 
4d, MS: m/z $520\left(\mathrm{M}^{+}\right)$; PMR: $\delta 9.78(\mathrm{~s}, 1 \mathrm{H})$, $7.81(\mathrm{~s}, 1 \mathrm{H}), 7.78-7.79(\mathrm{~m}, 1 \mathrm{H}), 7.48-7.49(\mathrm{~m}, 1 \mathrm{H}), 7.26-$ $7.37(\mathrm{~m}, 5 \mathrm{H}), 7.15-7.22(\mathrm{~m}, 2 \mathrm{H}), 2.35(\mathrm{~s}, 3 \mathrm{H}), 2.31$ (s, 3H),2.12 (s, 3H),2.06 (s, 3H); IR: 3107, 2951, 2920, 1685, 1598, 1120, 819, 786, $717 \mathrm{~cm}^{-1}$.

4e, MS: $m / z 647\left(\mathrm{M}^{+}\right)$; PMR: $\delta 10.42$ (s, $1 \mathrm{H}), 8.12(\mathrm{~s}, 1 \mathrm{H}), 7.92(\mathrm{~d}, 2 \mathrm{H}, J=8.5 \mathrm{~Hz}), 7.76$ (s, 1H),7.69-7.70 (m, 2H),7.62-7.64 (m, 5H),7.33 (d, 1H, J = $7 \mathrm{~Hz}), 7.23-7.25(\mathrm{~m}, 2 \mathrm{H}), 2.36$ (s, 3H), 2.09 (s, 3H); IR: 3132, 2925, 1678, 1600, 1596, 1130, $814,758 \mathrm{~cm}^{-1}$.

\section{RESULTS AND DISCUSSION}

Pyrazolone derivatives were condensed with various aldehydes to obtain the products 1,2 , 3 and 4. Compound 1a in its IR spectrum shows carbonyl frequency band at $1676 \mathrm{~cm}^{-1}$ of pyrazolone ring. PMR spectrum of the compound exhibited signals of propyl side chain, a triplet at $\delta 1.04$ due to methyl group, sextet for methylene at $\delta 1.77$ and triplet at $\delta 2.68$ for another methylene. Compound $2 \mathrm{~g}$, in its IR spectrum exhibited band at $1651 \mathrm{~cm}^{-1}$ for $\mathrm{C}=\mathrm{O}$ group. PMR spectrum showed singlet at $\delta 7.45$ for $1 \mathrm{H}$ due to olefinic proton and a doublet at $\delta 8.18$ for $1 \mathrm{H}$ with $J=5.6 \mathrm{~Hz}$ for pyridine ring proton. Compound $3 d$, exhibited a band due to carbonyl group at
$1681 \mathrm{~cm}^{-1}$. PMR spectrum of $3 \mathrm{~d}$ showed the signals at $\delta 1.01$ triplet for $3 \mathrm{H}$ with $J=7.4 \mathrm{~Hz}, \delta 1.75$ sextet for $2 \mathrm{H}$ with $J=7.4 \mathrm{~Hz}$ and $\delta 2.66$ triplet for $2 \mathrm{H}$ with $J=7.4 \mathrm{~Hz}$ corresponding to propyl group. Signals at $\delta 7.57$, dd, $1 \mathrm{H}, J=1.0$ \& $3.6 \mathrm{~Hz}, \delta 7.72 \mathrm{dd}, 1 \mathrm{H}, J=1.0 \& 5.1 \mathrm{~Hz}$ are due to thiophene ring. Olefinic proton is observed at $\delta 7.78$ as a singlet. Singlet at $\delta 9.74$ is due to proton on pyrazole ring. Compound $4 \mathrm{a}$, showed band due to carbonyl group at $1687 \mathrm{~cm}^{-1}$. PMR spectrum of the compound exhibited three singlets at $\delta 2.09,2.35$ and 2.45 due to three methyl groups. Singlets at $\delta 7.82$ and 10.23 are due to olefinic proton and pyrazole ring proton respectively.

\section{Antimicrobial activity}

The antimicrobial activity of synthesized compounds 1a-h, 2a-g, 3a-e and 4a-e was determined in vitro against three bacterial strains Bacillus subtilis, Escherichia coli, Salmonella typhi and two fungal strains Alternaria alternata and F. Oxisporum. The antimicrobial activity of compounds against test fungi and bacteria by using well diffusion technique. Ciprofloxacin was used as reference compound for bacteria and ketoconazole for fungi. All the experiments were performed in triplicate.

From seen activities for tested microorganisms are not observed for our compounds.

\section{REFERENCES}

1 Kullampalayam, K. S.; Aiyalu, R.; Palaniappan, S.; Wattamwar, P. P. Bioorg. Med. Chem.Lett. 2014, 24 (13), 2940-2944.

2 Evstropov, A. M.; Yavorovskaya, V. E.; Vorobev, E. S.; Khudonogova, Z. P.; Medvedeva, S. G; Filmonov, V. D.; Prishchep, T. P.; Saratikov, A. S.J. Pharma. Chem.1992, 26, 426-430.

3 Al-Haiza, M. A.; El-Assiery, S. A.; Sayed, G. H.Acta. Pharma. 2001, 51, 251-261.

4 Sivakumar, K. K.; Rajashekhran, A.; Bugreddy, S.Int. J. Res. Pharma. Chem.2012, 2, 327337.

5 Devnath, H. P.; Islam, M. R. Banladesh J. Pharmacol. 2010, 5, 30-33.

6 Ragab, F. A.; Abdel-Gawad, N. M.; Georgey, H. H.; Said, M. F. Chem. Pharma. Bull. 2013, 61(8), 834-845.
Pharma. Sci.2011, 1, 115-120.

8 Ye, D.; Zhang, Y.; Wang, F.; Zhang, M.; Zhang, X.; Jiang, H.; Liu, H. Bioorg. Med. Chem. 2010, 18, 1773-1782.

9 Bell, F. W.; Cantrell, A. S.; Hogberg, M.; Jaskunas, S. R.; Johansson, N. G.; Jordan, C. L.; Kinnick, M. D.; Lind, P.; Morin, J. R.; Noreen, R.; Oberg, B.; Palkowitz, J. A.; Parrish, C. A.; Pranc, P.; Sahlberg, C.; Ternansky, R. J.; Vasileff, R. T.; Vrang, L.; West, S. J; Zhang, H.; Zhou, X. X. J. Med. Chem. 1995,38, 49294936.

Patt, W. C.; Hamilton, H. W.; Taylor, M. D.; Ryan, M. J.; Taylor, Jr D. G.; Conolly, C. J. C.; Doherty, A. M.; Klutchko, S. R.; Sircar, I.; Steinbaugh, B. A.; Batley, B. L.; Painchaud, C. A.; Rapundalo, S. T.; Michniewicz, B. M.; Olson, S. C. J. Med. Chem. 1992,35, 2562- 
2572.

11

Rudolph, J.; Theis, H.; Hanke, R.; Endermann, R.; Johannsen, L.; Geschke, F. U. J. Med. Chem. 2001, 44, 619-626.

12 Yerragunta, V.; Suman, D.; Swamy, K.; Anusha, V.; Patil, P.; Naresh, M.PharmaTutor. 2014, 2(1), 40-48.

13 Mu, J-X.; Shi, Y-X.; Yang, M-Y.; Sun, Z-H.; Liu, X-H.; Li, B-J.; Sun, N-B.; Molecules, 2016, 21, 3-11.

14 Ouyang, G.; Chen, Z.; Cai, X-J.; Song,B-A.; Bhadury, P. S.; Yang, S.; Jin, L-H.; Xue, W.; Hu, D-Y.;Zeng,S.Bioorg. Med. Chem. 2008,16, 9699-9707.

15 Domiai, S.; El-Mallah, A.; Ghoneim, A.; Bekhit, A.; Razik, H. A. E. Inflammopharmacol.2016, 24, 163-172.

16 Kuettel, S.; Zambon, A.; Kaiser, M.; Burn, R.; Scapozza, L.; Perozzo, R. J. Med. Chem. 2007, 50, 5833-5839.

17 Farag, A. M.; Mayhoub A. S.; Barakat, S. E.; Bayomi, A.; H. Bioorg. Med. Chem. 2008, 16, 881-889.

18 Patel, N. B.; Agravt, S. N.; Shaikh, F. M. Med. Chem. Res. 2016, 20, 1033-1041.

19 Bassyouni, F. A.; Tawfik, H. A.; Soliman, A. M.; Rehim, M. A. Res. Chem.Intermed. 2012,38, 1291-1310.
20 Fadda, A .A.; Berghot, M. A.; Amer, F. A.; Badawy, D. S.; Bayoumy, N. M. Arch. Pharm. Chem. 2012, 345(5), 378-385.

21 Vijesh, A.M.; Isloor, A. M.; Telkar, S.;Arulmoli, T.; Fun, H-K.Arebian J. Chem. 2013, 6(2), 197-204.

22 Fascio, M. L.; Errea, M. I.; D’Accorso, N. B.Eur. J. Med. Chem. 2015, 90, 666-683.

23 Steenackers, H. P.; Levin, J. C.; Janssens, J. C.; Weerdt, A. D.; Balzarini, J.; Vanderleyden, J.; De Vos, D. E.; De Keersmaecker, S. C. Bioorg. Med. Chem. 2010, 18, 5224-5233.

24 Takate, S. J.; Karale, B. K.; Salve, S. P.; Zaware, B. H.; Jadhav, S. S. Orient. J. Chem. 2015,31(1), 307-315.

25 Zhang, Y.; Zheng, K.; Yan, H.; Jin, G.; Shao, C.; Zhou, X.; Zhou, Y.; He, T.2014, http://www. biomedcentral.com/1471-230X/14/62.

26 Sachdeva, H.; Dwivedi, D.; Goyal, P. Org. Chem. Int. 2013, Article ID 976032, http:// dx.doi.org/10.1155/2013/976032. Madje, B. R.; Shindalkar, S. S.; Ware, M. N. Shingare, M. S. Arkivoc(xiv) 2005, 82-86. Gadakh, A.; Pandit, C.; Rindhe, S. S.; Karale, B. K. Int. J. Chem. 2(2), 2010, 206-212.

29 Gadhave, A.; Kuchekar, S.; Karale, B. K. J. Chem. 2013, doi. org/10.1155/2013/741953. 